\title{
Jugular bulb and skull base pathologies: proposal for a novel classification system for jugular bulb positions and microsurgical implications
}

\author{
Sunil Manjila, MD, ${ }^{1}$ Timothy Bazil, BS, ${ }^{1}$ Matthew Kay, MBBS, ${ }^{2}$ Unni K. Udayasankar, MD, ${ }^{2}$ and \\ Maroun Semaan, MD $^{3}$ \\ 1Department of Neurosurgery, McLaren Bay Region Medical Center, Bay City, Michigan; '2Department of Medical Imaging, \\ University of Arizona College of Medicine, Tucson, Arizona; and ${ }^{3}$ Department of ENT, University Hospitals Cleveland Medical \\ Center, Cleveland, Ohio
}

OBJECTIVE There is no definitive or consensus classification system for the jugular bulb position that can be uniformly communicated between a radiologist, neurootologist, and neurosurgeon. A high-riding jugular bulb (HRJB) has been variably defined as a jugular bulb that rises to or above the level of the basal turn of the cochlea, within $2 \mathrm{~mm}$ of the internal auditory canal (IAC), or to the level of the superior tympanic annulus. Overall, there is a seeming lack of consensus, especially when MRI and/or CT are used for jugular bulb evaluation without a dedicated imaging study of the venous anatomy such as digital subtraction angiography or CT or MR venography.

METHODS A PubMed analysis of "jugular bulb" comprised of 1264 relevant articles were selected and analyzed specifically for an HRJB. A novel classification system based on preliminary skull base imaging using CT is proposed by the authors for conveying the anatomical location of the jugular bulb. This new classification includes the following types: type 1, no bulb; type 2, below the inferior margin of the posterior semicircular canal (SCC), subclassified as type 2a (without dehiscence into the middle ear) or type $2 b$ (with dehiscence into the middle ear); type 3 , between the inferior margin of the posterior SCC and the inferior margin of the IAC, subclassified as type $3 a$ (without dehiscence into the middle ear) and type $3 \mathrm{~b}$ (with dehiscence into the middle ear); type 4, above the inferior margin of the IAC, subclassified as type $4 \mathrm{a}$ (without dehiscence into the IAC) and type $4 \mathrm{~b}$ (with dehiscence into the IAC); and type 5, combination of dehiscences. Appropriate CT and MR images of the skull base were selected to validate the criteria and further demonstrated using 3D reconstruction of DICOM files. The microsurgical significance of the proposed classification is evaluated with reference to specific skull base/posterior fossa pathologies.

RESULTS The authors validated the role of a novel classification of jugular bulb location that can help effective communication between providers treating skull base lesions. Effective utilization of the above grading system can help plan surgical procedures and anticipate complications.

CONCLUSIONS The authors have proposed a novel anatomical/radiological classification system for jugular bulb location with respect to surgical implications. This classification can help surgeons in complication avoidance and management when addressing HRJBs.

https://thejns.org/doi/abs/10.3171/2018.5.FOCUS18106

KEYWORDS jugular bulb; skull base; jugular foramen; dehiscence; diverticulum; semicircular canal; endovascular management; endolymphatic sac

$\mathrm{T}$ $\checkmark$ HE jugular bulb that receives drainage from both intracranial and extracranial compartments is located posterolaterally within the pars vascularis of the jugular foramen..$^{22,26}$ The sigmoid sinus and inferior petrosal sinus drain into the jugular bulb, which then becomes the internal jugular vein (IJV) at the base of the skull. The tributaries of the jugular vein include: the middle thyroid vein, superior thyroid vein, lingual vein, facial vein, pharyngeal vein, and inferior petrosal sinus, apart from the vertebral venous plexus, venous plexus of the hypoglossal

ABBREVIATIONS CPA = cerebellopontine angle; HRCT = high-resolution CT; HRJB = high-riding jugular bulb; IAC = internal auditory canal; IJV = internal jugular vein; SCC = semicircular canal.

SUBMITTED March 2, 2018. ACCEPTED May 3, 2018.

INCLUDE WHEN CITING DOI: 10.3171/2018.5.FOCUS18106. 

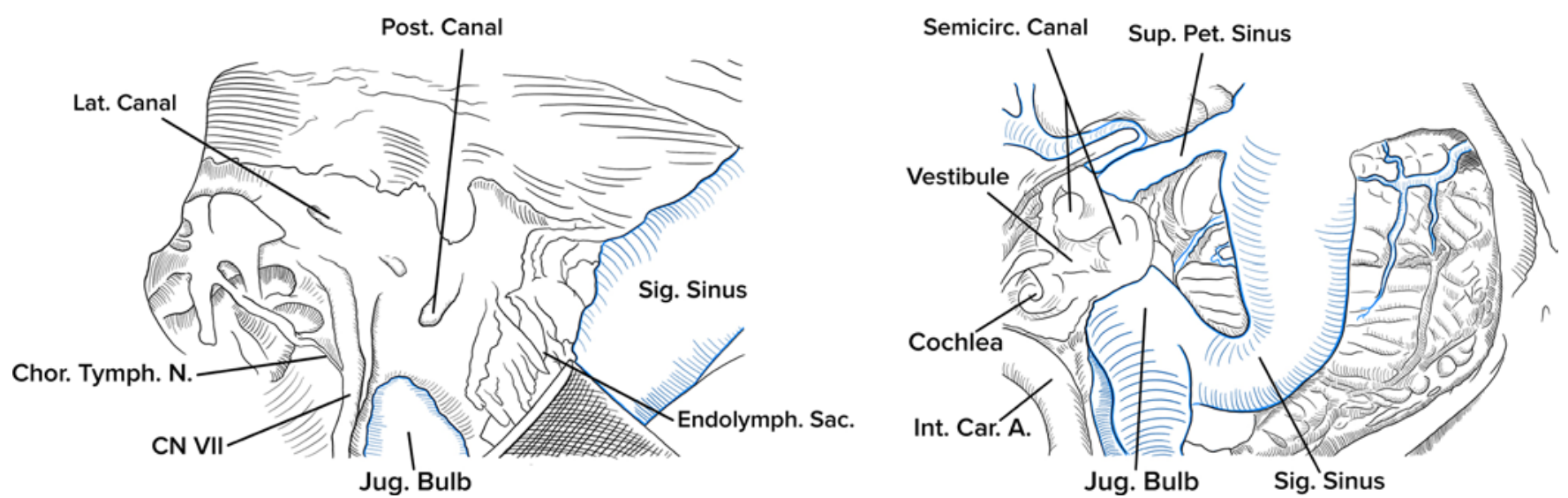

FIG. 1. Microsurgical anatomy showing the relationship of the jugular bulb (Jug. Bulb) to semicircular canals (Semicirc. Canal), cochlea, facial nerve (CN VII), and endolymphatic sac (Endolymph. Sac.). Chor. Tymph. N. = chorda tympani nerve; Int. Car. A. = internal carotid artery; Lat. Canal = lateral canal; Post. Canal = posterior canal; Sig. Sinus = sigmoid sinus; Sup. Pet. Sinus = superior petrosal sinus. Copyright Sunil Manjila. Published with permission.

canal, posterior condylar emissary vein, and veins along the petroclival fissure. ${ }^{26}$ Anteriorly the jugular bulb is limited by the internal carotid artery, cochlear aqueduct, inferior petrosal sinus, meningeal branch of the ascending pharyngeal artery, lower cranial nerves, and posterior meningeal artery. The posterior limits of the jugular bulb include the sigmoid sinus, occipital bone, and facial nerve, while the superior limits of the jugular bulb include the external auditory canal, middle ear, posterior semicircular canal (SCC), vestibule, and internal auditory canal (IAC; Fig. 1).

The upper limit of the jugular bulb is commonly found under the hypotympanum within the middle-ear cavity, ${ }^{14,35}$ and an atypical presentation of the jugular bulb may be visualized as an upward extension of the bulb that invades into the hypotympanum. ${ }^{35}$ Sasindran et al. defines this extension of jugular bulb presenting in the middle-ear space with a thin or nonexistent bony septum as a high-riding jugular bulb (HRJB), which has been previously subclassified as "with dehiscence" or "without dehiscence." ${ }^{35}$ An alternate definition of an HRJB has been proposed when it is observed above the tympanic annulus or no greater than $2 \mathrm{~mm}$ from the IAC. ${ }^{35}$ Singla et al. postulated the jugular bulb as high riding when the distance of the summit of the jugular fossa from the round window or IAC was less than or equal to $2 \mathrm{~mm}$ or if there is no distance between the jugular fossa and the slit on which the endolymphatic sac opens. ${ }^{40}$

MRI offers multiplanar sequences of skull base anatomy that can help the visualization of HRJBs (Fig. 2). However, high-resolution CT (HRCT) is considered the best imaging modality for evaluation of HRJB. Couloigner et al. has compiled different iterations of diagnosing HRJB based on temporal bone imaging where high location is implicated when the bulb reaches 1) the inferior part of round window, 2) the inferior part of the sulcus tympani, 3) the basal turn of the cochlea, and 4) $2 \mathrm{~mm}$ under the inferior edge of the IAC. 7 Shao et al. classified jugular bulbs as 1) grade 1, jugular bulb located less than $1 \mathrm{~mm}$ above the lower border of IAC; 2 ) grade 2 , jugular bulb between 1.5 and $3 \mathrm{~mm}$ above the lower border of the IAC; and 3) grade
3 , jugular bulb greater than $3 \mathrm{~mm}$ above the lower border of the IAC. ${ }^{37}$ We believe that these anatomical boundaries of the jugular bulb and extent of the bulb itself are not evolving forms, and hence the term "classification" seems to be more appropriate than "grading."

Park et al. subclassified HRJB into two types based on axial CT images: type 1, in which the bulb dome reaches above the inferior part of the round window; and type 2 , when the dome is higher than the inferior edge of the IAC. ${ }^{28,29}$ It is apparent that there is no consensus on the exact definition of HRJB, and multiplanar structures that define the critical microsurgical boundaries (SCC, IAC, round window, and endolymphatic sac) of the skull base cannot often be analyzed based only on limited standard axial CT sections of temporal bone, without reconstruction.

The current proposal of the Manjila and Semaan classification accounts for the relationship of the IAC, posterior SCC, and presence or absence of dehiscences into the middle ear or IAC (Fig. 3). This straightforward and practical classification system with easy subcategorization based on local skull base landmarks would be extremely useful in preoperative planning of surgical corridors and thus achieve satisfactory clinical outcomes. This classification can be readily applied to a wide cohort of cases such as vestibular and jugular foramen schwannomas, giant cholesteatomas, and cochlear implants, as well as endovenous stent/coiling procedures to overcome some of the inherent technical limitations.

In preparation of this paper, the relevant articles were retrieved from the PubMed electronic database. Of 1264 publications on the jugular bulb as of February 27, 2017, 738 articles discussed surgery of the bulb, and 22 of them reported HRJBs. Jugular bulb dehiscences were studied in 57 articles and diverticula in 51 articles. The articles discussing surgery of the jugular bulb were extracted and the data assessed for quality by two reviewers.

\section{Development of the Jugular Bulb}

The jugular bulb develops during childhood, particularly when the child has gained the ability to stay upright 

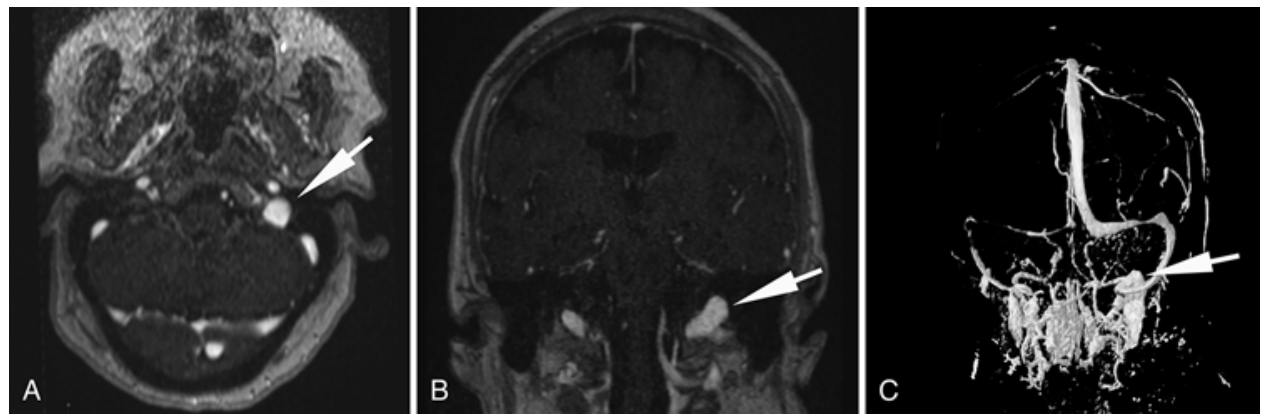

FIG. 2. Postcontrast (gadobenate dimeglumine) MR venography in the axial (A) and coronal (B) planes with the corresponding 3D contrast MR venogram (C) of a patient with a Manjila and Semaan type 2a jugular bulb (arrow).

around 2 years of age. ${ }^{11,25,27,28}$ The jugular bulb continues to develop through childhood and becomes stable in adulthood. Once an erect posture is attained in life, the ascending negative pulse waves originating from the right atrium are postulated to be transmitted rostrally into the jugular sinus leading to the dilation or formation of the jugular bulb. ${ }^{28}$ Consequently, an HRJB is also considered a risk factor for jugular bulb dehiscence. ${ }^{12,28}$ Because there is growth and plateauing of jugular bulb development with age, there is debate on the role of temporal bone pneumatization and the orientation of SCCs in determining the location of the jugular bulb. Of note, Lee et al. analyzed CT images of 84 patients for petrous apex pneumatization and reported that the pneumatization process in the petrous part of temporal bone is independent of other parts of temporal bone and it may not be influenced by major temporal bone structures. ${ }^{19}$ There are theories that the mastoid pneumatization partly determines the location of the jugular bulb; the distance from the external auditory canal to the sigmoid sinus appear to be relatively shorter in cases of little or absent pneumatization. ${ }^{28}$ Likewise, with age, SCC orientation changes may also occur. Lyu et al. reported that SCCs have a tendency to tilt anteriorly simultaneously as a whole with age, where the jugular bulb position, especially the anteroposterior position, appears to be correlated with the orientation of SCCs. ${ }^{20}$

An HRJB has been associated with the occurrence of Ménière's disease or endolymphatic hydrops in several cases, in which a dehiscence with the inner ear is proposed as the etiology. $3,5,7,13,31$ Symptoms resulting from an HRJB vary and can often be effectively managed medically. While an HRJB may also be asymptomatic, it appears to produce clinical symptoms such as tinnitus, vertigo, dizziness, and hearing loss. ${ }^{1,2,10,31,35}$

\section{Jugular Bulb Distension, Diverticulum, Dehiscence, and Pseudolesions}

The etiology of jugular bulb anomalies is poorly understood, and the pathophysiology of diverticulum formation is relatively unknown. It has been suggested that abnormal venous flow, whether it is hypertension or turbulence, is a contributing factor. ${ }^{28}$ Venous flow patterns have been analyzed in the jugular veins, and Kao et al. demonstrated a distinctive helical flow pattern in the proximal IJV, when jugular bulbs are elevated. ${ }^{15}$ The role of jugular bulb ge- ometry in the exact causation of pulsatile tinnitus is still debated. ${ }^{4}$ In the context of turbulent and slow venous flow, there are often increased signal intensities noted on MRI (in particular T1-weighted images), especially on the side with large or HRJBs simulating an intraluminal mass. A contrast CT venogram, phase-contrast MR venogram, or even careful analysis of non-T1-weighted sequences can exclude an intraluminal "pseudolesion" of the jugular bulb.

An HRJB may be visualized otoscopically as a blue mass behind the tympanic membrane, and a Valsalva maneuver or ipsilateral jugular compression can cause distension of the jugular bulb. ${ }^{35}$ When the sigmoid plate is absent, the bulb may protrude into the middle-ear cavity, which is classified as a dehiscent jugular bulb. ${ }^{29,33}$

A jugular bulb diverticulum is an outpouching from an HRJB, which is debated to be either a true venous anomaly or a variant of a medially located HRJB. It cannot be visualized otoscopically because it does not extend into the middle ear, so it is diagnosed by radiography.

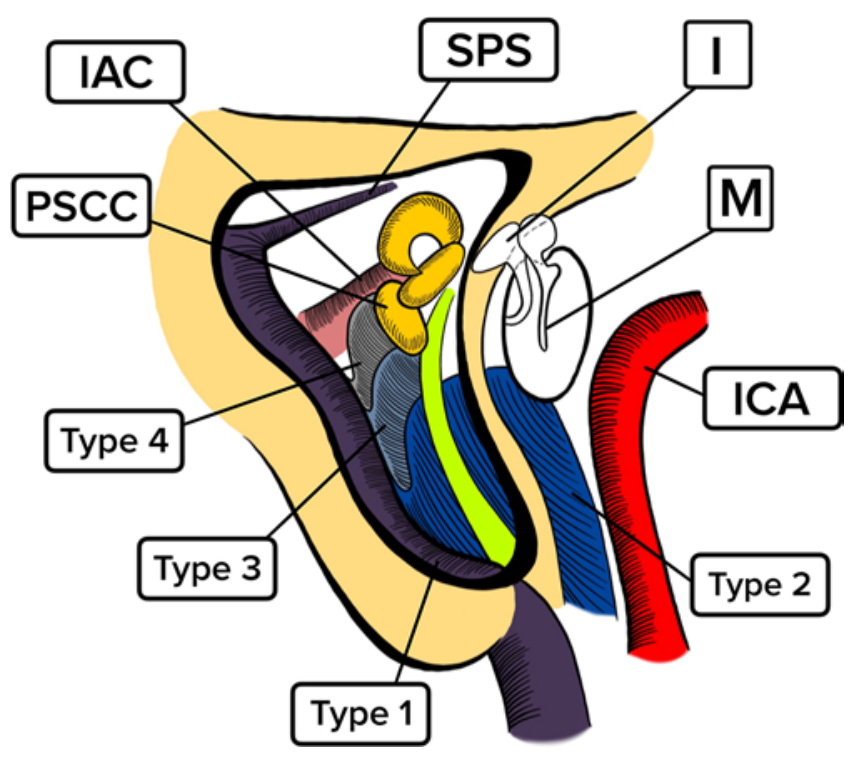

FIG. 3. Schematic representation of the Manjila and Semaan classification of the various jugular bulb types/locations with respect to anatomical landmarks, including the IAC, superior petrosal sinus (SPS), posterior SCC (PSCC), incus (I), malleus (M), and internal carotid artery (ICA). Copyright Sunil Manjila. Published with permission. 
TABLE 1. Manjila and Semaan classification of jugular bulb location

\begin{tabular}{cl}
\hline Type 1 & No jugular bulb \\
\hline Type 2 & Below inferior margin of posterior SCC \\
\hline Type 2a & Without dehiscence into the middle ear \\
\hline Type 2b & With dehiscence into the middle ear \\
\hline Type 3 & $\begin{array}{c}\text { Between inferior margin of posterior SCC \& inferior } \\
\text { margin of IAC }\end{array}$ \\
\hline Type 3a & Without dehiscence into the middle ear \\
\hline Type 3b & With dehiscence into the middle ear \\
\hline Type 4 & Above inferior margin of IAC \\
\hline Type 4a & Without dehiscence into the IAC \\
\hline Type 4b & With dehiscence into the IAC \\
\hline Type 5 & Combination of dehiscences \\
\hline
\end{tabular}

Often this is an incidental finding but some patients present with sensorineural hearing loss from dehiscence into the IAC or posterior SCC, or Ménière's syndrome from encroachment into the endolymphatic sac..$^{16,36,41,43}$ Jugular bulb diverticulum is best seen on HRCT or CT as a wellcorticated polypoid projection from the jugular bulb itself, commonly projecting superomedially into the petrous bone medial to the labyrinth and partly covered by the dense otic capsule. HRJBs can pose some surgical challenges when a transotic approach is planned for treating skull base pathologies.

\section{New Classification and Surgical Relevance of Jugular Bulb Anomalies}

We propose a new CT scan-based classification for jugular bulb location: 1) type 1, no bulb; 2) type 2, below the inferior margin of the posterior SCC, subclassified as type $2 \mathrm{a}$ (without dehiscence into the middle ear) or type $2 \mathrm{~b}$ (with dehiscence into the middle ear; 3 ) type 3, between the inferior margin of the posterior SCC and inferior margin of the IAC, subclassified as type $3 \mathrm{a}$ (without dehiscence into the middle ear) or type $3 \mathrm{~b}$ (with dehiscence into the middle ear); 4) type 4 , above the inferior margin of the IAC, subclassified as type $4 \mathrm{a}$ (without dehiscence into the IAC) or type $4 \mathrm{~b}$ (with dehiscence into the IAC); and 5) type 5, combination of dehiscences (Table 1). We have selected these criteria keeping in mind the clinical implications of both microsurgical and endovascular interventions in various otologic/neurosurgical conditions (Figs. 3-6).

We have made appropriate CT and MR images of the skull base to elucidate the criteria for classification and further demonstrated these using 3D reconstruction of DICOM files (Fig. 6). High spatial resolution CT patient data of representative HRJB classifications were acquired for visualization of key anatomical structures. Segmentation software (Materialise Mimics 21) was used to model the anatomy for each patient. Following the import of each CT scan into the software, automatic segmentation tools produced a 3D model of the bony skull. The IAC, SCC, and jugular vein were manually segmented slice by slice in the software. Segmented anatomies were exported as STL (Standard Tessellation Language) files. Freeform from 3DSystems performed further refinements to the 3D models as needed, then produced images with transparent bone anatomy and colored IACs, SCCs, and jugular veins. For video production, stereolithographs from one patient were exported from Freeform to a .bip format to be used with Luxion Keyshot 5. Rotations were prescribed to the parts with Keyshot, then rendered to produce a video centered around the pertinent anatomies. Video reconstructions were created to understand the $360^{\circ}$ rotational anatomy from the perspective of various posterior and posterolateral skull base approaches to jugular foramen lesions and anterior cerebellopontine angle (CPA) lesions (Video 1).

VIDEO 1. Clip showing rotational view of 3D reconstruction of CT temporal bone images demonstrating the position of bilateral jugular bulbs in each case, with respect to the posterior SCC and IAC. Copyright Sunil Manjila. Published with permission. Click here to view.

\section{Posterior Fossa Microsurgery and HRJBs}

There are several microsurgical techniques and landmarks for safe exposure of the jugular foramen and its contents in lateral/far-lateral surgical approaches to the skull base.

CPA tumors (vestibular or nonvestibular schwannomas and meningiomas) are the most common extraaxial lesions of the posterior fossa. ${ }^{42}$ Acoustic schwannomas that arise from the CPA will usually present with highfrequency hearing loss. Nonvestibular schwannomas, however, will have predominating cerebellar symptoms and other cranial nerve deficits. Imaging characteristics are often diagnostic of these lesions, and may also demonstrate the presence and location of an HRJB. Treatment often includes microsurgical resection of the tumor with attempt to preserve facial nerve function, but observation or subtotal resection may alternatively be entertained depending on the extent of hearing loss, facial nerve function, and systemic medical status of the patient. ${ }^{42}$ The standard treatment for CPA meningiomas is microsurgical excision with the objective of complete resection of the tumor, preferably including that of involved dura and hyperostotic bone depending on the anatomical extent of the lesion and involvement (encasement vs displacement) of adjacent neurovascular structures. Alternative options include stereotactic radiotherapy or open craniotomy with preoperative embolization, and the multimodality of treatment offered to the patient depends on the experience of the surgeon, institutional protocols, and patient preference. Complications of a retromastoid suboccipital approach to the CPA include hearing loss (most common), injury to the anterior inferior cerebellar artery or its labyrinthine branch (resulting in hemorrhage/ischemia and hearing loss, respectively), cerebellar retraction injury, facial paresis with or without vestibular dysfunction, lower cranial nerve and brainstem injury, and hydrocephalus, apart from wound-related issues like postoperative intracranial bleeds, CSF leak, and pseudomeningocele. Among the above-listed morbidities from a retromastoid suboccipital approach, only the drilling of the IAC would accentuate the risk of hemorrhage or facial paresis with or without vestibular dysfunction in an HRJB. ${ }^{42}$ 

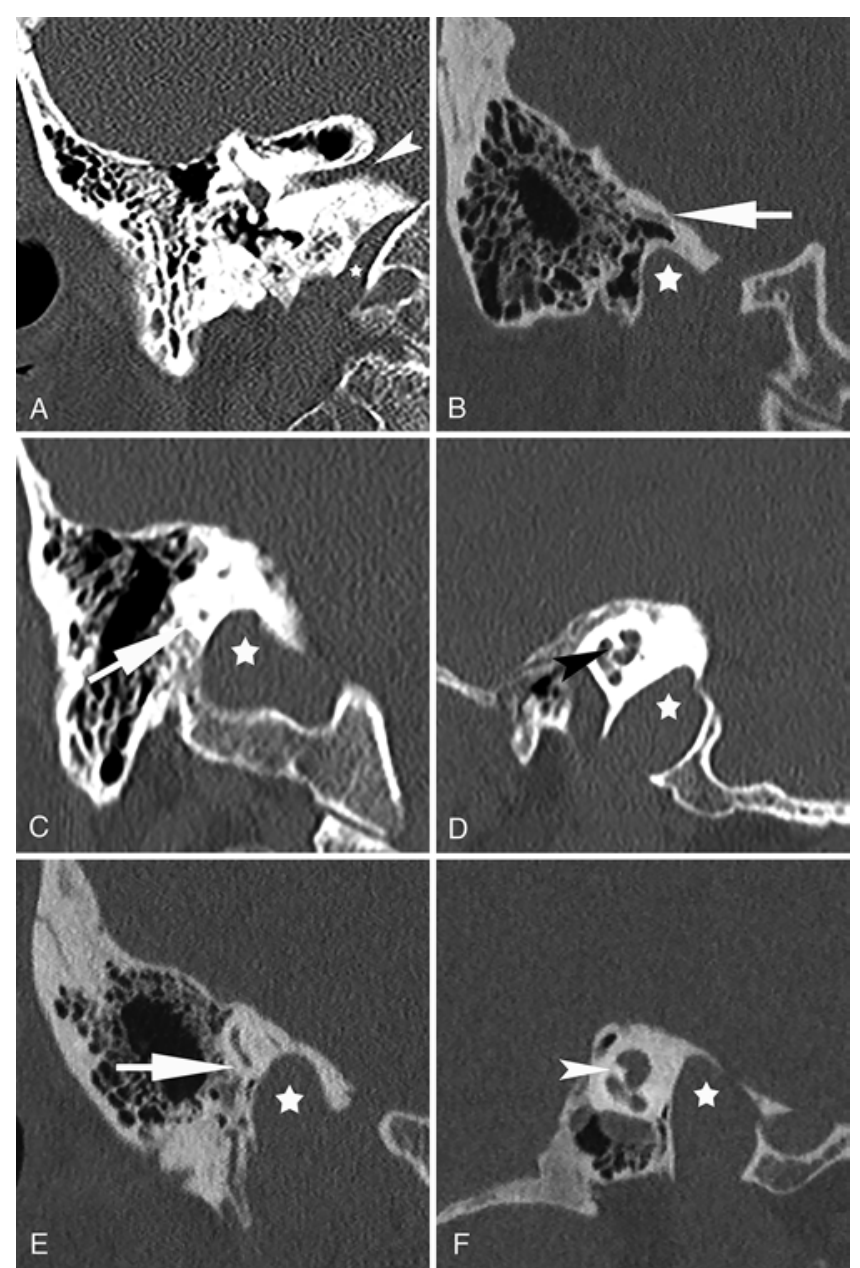

FIG. 4. Manjila and Semaan classification for each jugular bulb location/ type on the right temporal bone CT scans of various patients. Type 1 is demonstrated in the coronal plane $(\mathbf{A})$ with no jugular bulb and the condylar canal (star), containing the occipital emissary vein, receives drainage from the sigmoid sinus, which is referenced with respect to the IAC (arrowhead). Type 2 is demonstrated in the coronal plane (B) with the jugular bulb (star) inferior to the posterior SCC (arrow). Type 3 is demonstrated in the coronal (C) and sagittal (D) planes with the jugular bulb (star) above the inferior margin of the posterior SCC (arrow) but below the inferior margin of the IAC, which is imaged at the fundus with the modiolus (arrowhead). Type 4 is demonstrated in the coronal $(E)$ and sagittal $(F)$ planes with the jugular bulb (star) well above the inferior margin of the posterior SCC (arrow) as well as the inferior margin of the IAC, which is imaged at the fundus with the modiolus (arrowhead). None of the jugular bulbs in $\mathrm{B}-\mathrm{F}$ demonstrate dehiscence into the middle ear $(B-D)$ nor dehiscence into the IAC ( $E$ or $F$ ) and are therefore considered type $2 \mathrm{a}(\mathrm{B})$, type $3 \mathrm{a}(\mathrm{C}$ and $\mathrm{D})$, and type $4 \mathrm{a}(\mathrm{E}$ and $\mathrm{F})$ jugular bulbs.

An HRJB, if encountered in the translabyrinthine approach of acoustic schwannomas, can be exposed after extensive bone decortication and decompressed during surgery by pressure using Gelfoam and cottonoids. ${ }^{23}$ The relationship of an HRJB in a transotic approach is critical as it can hamper the near-circumferential exposure of the IAC in the latter. ${ }^{46} \mathrm{HRJBs}$ can make endoscopic access to the IAC very difficult in certain cases, just like in other skull base approaches to the region. . $^{21,30,34}$

We feel that the currently proposed classification can offer safer and more efficacious surgical planning to the
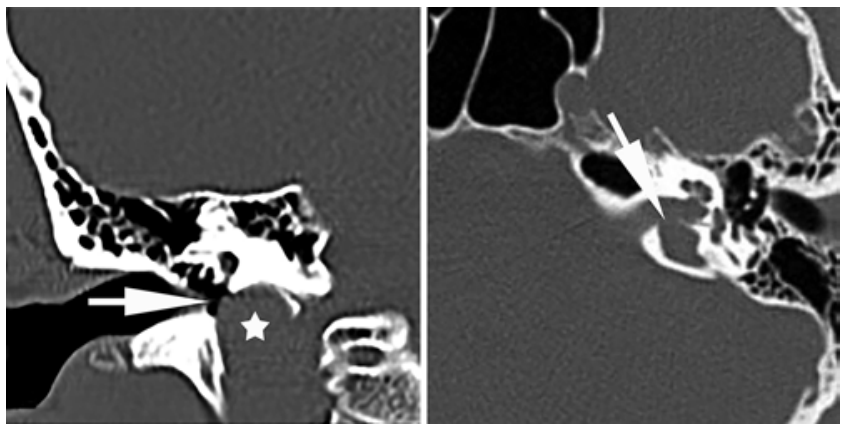

FIG. 5. Left: Right temporal bone CT in the coronal plane for a patient with a Manjila and Semaan type $3 \mathrm{~b}$ jugular bulb because there is jugular bulb (star) dehiscence into the middle ear (arrow). The jugular bulb is above the inferior margin of the posterior SCC but below the inferior margin of the IAC (not demonstrated). Right: Left temporal bone CT in the axial plane for a patient with a Manjila and Semaan type $4 \mathrm{~b}$ jugular bulb because there is jugular bulb dehiscence into the IAC (arrow). Modified with permission from Park et al: Korean J Audiol 16:39-42, 2012. Copyright (2012) by the Korean Audiology Society.

described region. In Manjila and Semaan classification types 1 and 2, a transpetrous approach to the posterior fossa (translabyrinthine approach) provides adequate exposure given adequate skeletonization of the sigmoid sinus and dome of the jugular bulb is performed. In types 3 and 4 , the exposure is hindered by the height of the bulb. The risk of injuring the jugular bulb in increased in a transpetrous approach in type 3 and 4 bulbs. The retrosigmoid approach can provide a wider exposure to the CPA, but the risk of injuring the jugular bulb is increased in type 3 and 4 bulbs during removal or drilling of the posterior lip of the IAC to expose the lateral two-thirds of the IAC.

Jugular foramen schwannomas are another subset of skull base lesions in which an HRJB can be a surgical risk for intraoperative hemorrhage. ${ }^{17}$ The jugular foramen can be accessed via many approaches, including a postauricular transtemporal approach, retrosigmoid approach, far-lateral approach, and preauricular subtemporal-infratemporal approach, to name a few. There are many microsurgical modifications and endoscope-assisted options described in the neurosurgical literature. There can also be torrential venous hemorrhage from inadvertent injury of an HRJB in these operations. The potential surgical option for intraoperative management includes a firm packing of the jugular bulb with possible clipping of the sigmoid sinus. The sigmoid sinus can also be taken down with a ligature introduced using an aneurysm passer. However, there were concerns of developing iatrogenic cranial neuropathies from closing down the inferior petrosal sinus inflow. An endovascular transvenous intervention may be considered a minimally invasive option if readily available and a patient can be maintained hemodynamically stable for the embolization procedure.

Of many clinical pathologies, a glomus jugulare tumor is an uncommon pathology of the skull base that can prove challenging to treat. ${ }^{9}$ While observation is a treatment option, interventional modalities include resection, endovascular embolization, and stereotactic radiation therapy, or a combination of these 3 methods. ${ }^{9}$ Combination therapy 


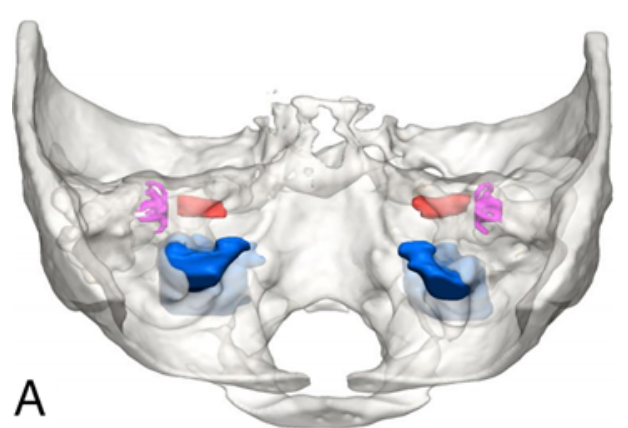

L

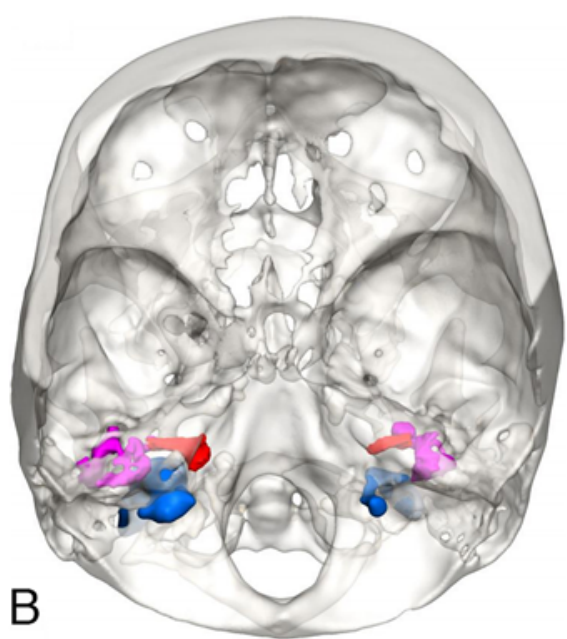

$\mathrm{L}$

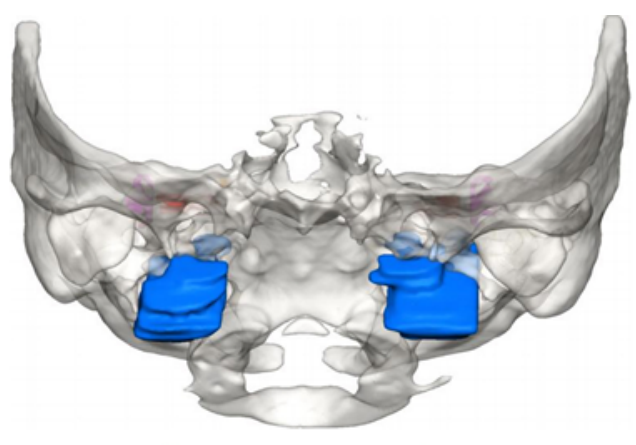

$\mathrm{R}$

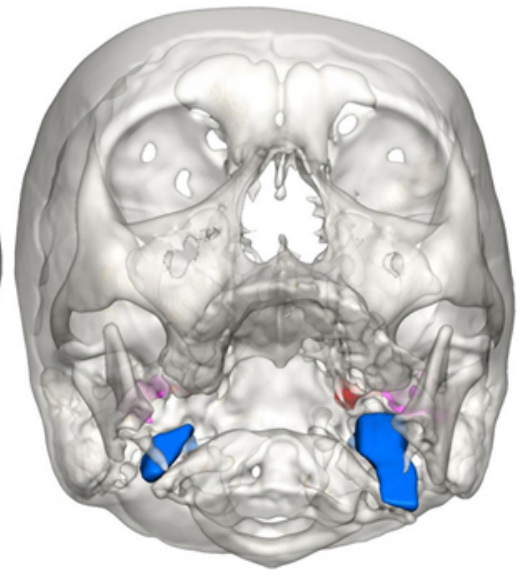

$\mathrm{R}$

L

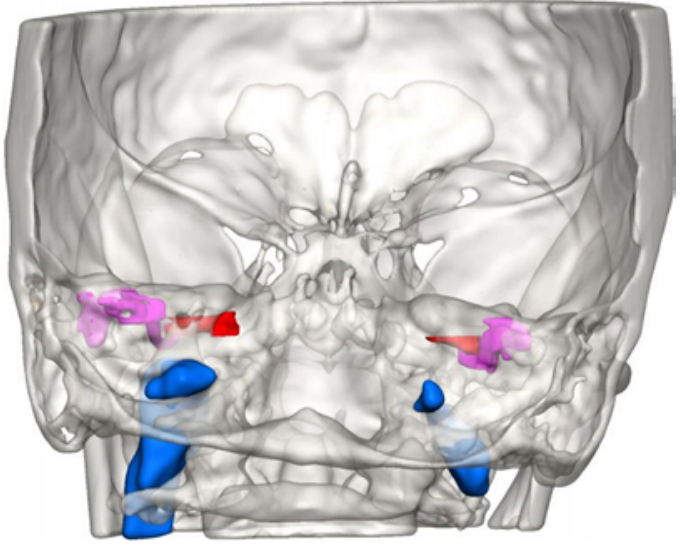

L

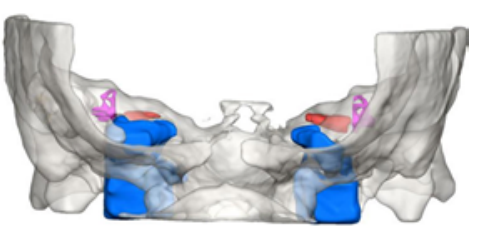

$\mathrm{L}$

$\mathrm{R}$

FIG. 6. DICOM data sets were utilized from the temporal bone CT scans of 2 different patients to produce 3D images to localize the jugular bulbs (blue) with respect to the posterior SCC of the inner ear (pink) and the IAC (red). The first patient (A) has right and left type $2 a$ jugular bulbs. The second patient (B) has a right type 1 and a left type 4 a jugular bulb.

is likely the best option for patients with preserved cranial nerve function, although the decision-making algorithms are more complex and debatable. When complete tumor resection with preserved function is achievable, surgery is considered the best treatment option. ${ }^{9}$ The surgeon should be aware of any underlying jugular bulb anomalies, bone erosions, additional venous tributaries, and venous outflow channels prior to surgical intervention, regardless of a specific approach taken when operating on glomus jugulare tumors. The patient's unique presentation, age, medical history, tumor characteristics, and lower cranial nerve status are important in evaluating the treatment plan. ${ }^{9}$ Endovascular anatomy of the jugular bulb and its tributaries plays an important role in the clinicoradiological outcome of staged treatment of these lesions. ${ }^{47}$

HRJB is not per se a contraindication for middle-ear surgery, however, there are reports of inadvertent intraoperative injuries and being aware preoperatively may minimize this risk. ${ }^{32}$ The surgeon should be mindful of an HRJB when it is identified preoperatively, as these patients are at greater risk for complications during middle-ear surgery than patients with normal anatomy. The management of symptomatic HRJB is usually conservative and medical (labyrinthine sedatives, control of systemic hypertension, etc.), although there are cases of surgical management if severe symptoms such as vertigo, hearing loss, or tinnitus are present. ${ }^{45}$ One surgical option is jugular-vein ligation, which has shown successful outcomes, but there is an associated risk of idiopathic intracranial hypertension. The surgical management of jugular bulb dehiscence comprises mastoidectomy and impaction of bone wax, with the risk of injury to the facial nerve, middle ear, and dura mater. Dehiscent bulbs can also be resurfaced using hydroxyapatite cement. ${ }^{8}$

Preoperative awareness of HRJB is also important in patients with cochlear implants as well as resection of giant cholesteatomas of the mastoid that require combined surgical approaches. ${ }^{6,18,45}$ Miller et al. described the usefulness of decompressing and retracting the jugular bulb to improve infralabyrinthine drainage of cholesterol granulomas. ${ }^{23}$ They also demonstrated surgical techniques for jugular bulb decompression and retraction to expand infralabyrinthine access for the biopsy and gain access to petroclival tumors with minimal to no intracranial extension. ${ }^{23}$

\section{Endovascular Management: Significance of High Jugular Bulb, Dehiscence, and Diverticula}

The etiology of pulsatile tinnitus is described in the literature in the descending order of HRJB, dehiscent bulbs, 
and jugular bulb diverticula. Trivelato et al. described a case of jugular bulb diverticulum associated with transverse-sigmoid sinus stenosis, presenting with pulsatile tinnitus. ${ }^{44}$ The patient underwent dural sinus stent placement and selective embolization of the diverticulum with good results. Mortimer et al. reported a case of a 58-year-old woman with two wide-necked jugular bulb diverticula (which extended superiorly and laterally) that showed resolution of pulsatile tinnitus at 10 months' follow-up after 2-stage procedures. ${ }^{24}$ Using a transfemoral approach they catheterized the right IJV and a self-expandable Luminexx stent (Bard Peripheral Vascular, Inc.) was deployed, and then Penumbra microcoils were used to treat the superior diverticulum with initial partial symptomatic relief. At 5 months the superior diverticulum was found to be thrombosed on the repeat catheter venogram. The partially filling lateral diverticulum was then treated with multiple coils and the patient subsequently became asymptomatic. Signorelli et al. demonstrated endovascular treatment of two concomitant cases of pulsatile tinnitus involving sigmoid sinus stenosis and ipsilateral jugular bulb diverticula. ${ }^{39}$ Shihada et al. described endovascular embolization of a hemorrhagic jugular bulb diverticulum. ${ }^{38}$

The proposed novel classification of jugular bulb positions would help predict anatomical location of likely hemorrhage in a given surgical procedure or approach where it is likely to be injured. The new Manjila and Semaan classification has a $3 \mathrm{D}$ perspective that allows better planning of endovascular transvenous intervention as in the case of jugular venous stenting, especially with increasing use of flat-panel CT (DynaCT, Siemens) in postoperative followup. Incorporating osseous anatomy into jugular transvenous procedures is akin to corroboration of angiographic segments of intracranial carotid artery into the planning of transarterial stent deployment.

\section{Conclusions}

The authors have proposed a novel anatomical/radiological classification system for jugular bulb location. The close anatomical relationships of internal acoustic meatus and SCCs were recapitulated and validated by artistic rendition and 3D reconstruction of DICOM data from representative patient samples. We believe that this classification system will help skull base surgeons in the choice of surgical approaches, complication avoidance, and management, as well as addressing HRJBs, dehiscence, or diverticula adjacent to the surgical corridors. Additionally, this novel classification has a 3D rotational perspective that can also allow better planning of endovascular transvenous interventions, such as in jugular venous stenting across the skull base.

\section{Acknowledgments}

We acknowledge the assistance of Travis Simpson (Lead Engineer, KLS Martin Manufacturing LLC) for the 3D reconstruction of images and Patti Wesenick (librarian, McLaren Bay Region Medical Center, Bay City, Michigan) for making this paper possible.

\section{References}

1. Barr JG, Singh PK: A rare cause of conductive hearing loss: high lateralized jugular bulb with bony dehiscence. Ear Nose Throat J 95:227-229, 2016

2. Bektas D, Caylan R: Non-pulsatile subjective tinnitus without hearing loss may be caused by undetectable sounds originating from venous system of the brain. Med Hypotheses 71:245-248, 2008

3. Bilgen C, Kirazli T, Ogut F, Totan S: Jugular bulb diverticula: clinical and radiologic aspects. Otolaryngol Head Neck Surg 128:382-386, 2003

4. Brook CD, Buch K, Gadaleta D, Jalisi S, Grillone G, Sakai O, et al: Changes in the jugular bulb associated with sacrifice of the internal jugular vein. Otol Neurotol 36:146-150, 2015

5. Brook CD, Buch K, Kaufmann M, Sakai O, Devaiah AK: The prevalence of high-riding jugular bulb in patients with suspected endolymphatic hydrops. J Neurol Surg B Skull Base 76:471-474, 2015

6. Cazzador D, Favaretto N, Zanoletti E, Martini A: Combined surgical approach to giant cholesteatoma: a case report and literature review. Ann Otol Rhinol Laryngol 125:687-693, 2016

7. Couloigner V, Grayeli AB, Bouccara D, Julien N, Sterkers O: Surgical treatment of the high jugular bulb in patients with Ménière's disease and pulsatile tinnitus. Eur Arch Otorhinolaryngol 256:224-229, 1999

8. DeHart AN, Shaia WT, Coelho DH: Hydroxyapatite cement resurfacing the dehiscent jugular bulb: novel treatment for pulsatile tinnitus. Laryngoscope 128:1186-1190, 2018

9. Fayad JN, Schwartz MS, Brackmann DE: Treatment of recurrent and residual glomus jugulare tumors. Skull Base 19:92-98, 2009

10. Fox R, Nash R, Tatla T: Encountering a high jugular bulb during ear surgery. Ann R Coll Surg Engl 99:36-37, 2017

11. Friedmann DR, Eubig J, McGill M, Babb JS, Pramanik BK, Lalwani AK: Development of the jugular bulb: a radiologic study. Otol Neurotol 32:1389-1395, 2011

12. Friedmann DR, Eubig J, Winata LS, Pramanik BK, Merchant SN, Lalwani AK: Prevalence of jugular bulb abnormalities and resultant inner ear dehiscence: a histopathologic and radiologic study. Otolaryngol Head Neck Surg 147:750-756, 2012

13. Gopen Q, Zhou G, Poe D, Kenna M, Jones D: Posterior semicircular canal dehiscence: first reported case series. Otol Neurotol 31:339-344, 2010

14. Inal M, Muluk NB, Dağ E, Arıkan OK, Kara SA: The pitfalls and important distances in temporal bone HRCT of the subjects with high jugular bulbs - preliminary report. Adv Clin Exp Med 24:315-324, 2015

15. Kao E, Kefayati S, Amans MR, Faraji F, Ballweber M, Halbach V, et al: Flow patterns in the jugular veins of pulsatile tinnitus patients. J Biomech 52:61-67, 2017

16. Karatas A, Kocak A, Cebi IT, Salviz M: Comparison of endolymphatic duct dimensions and jugular bulb abnormalities between meniere disease and a normal population. J Craniofac Surg 27:e424-e426, 2016

17. Komune N, Matsushima K, Matsushima T, Komune S, Rhoton AL Jr: Surgical approaches to jugular foramen schwannomas: an anatomic study. Head Neck 38 (Suppl 1):E1041E1053, 2016

18. Konishi M, Iwai H, Tomoda K: Reexamination of etiology and surgical outcome in patient with advanced external auditory canal cholesteatoma. Otol Neurotol 37:728-734, 2016

19. Lee DH, Kim MJ, Lee S, Choi H: Anatomical factors influencing pneumatization of the petrous apex. Clin Exp Otorhinolaryngol 8:339-344, 2015

20. Lyu HY, Chen KG, Yin DM, Hong J, Yang L, Zhang TY, et al: The age-related orientational changes of human semicircular canals. Clin Exp Otorhinolaryngol 9:109-115, 2016

21. Master A, Hamiter M, Cosetti M: Defining the limits of endoscopic access to internal auditory canal. J Int Adv Otol 12:298-302, 2016 
22. Matsushima K, Funaki T, Komune N, Kiyosue H, Kawashima M, Rhoton AL Jr: Microsurgical anatomy of the lateral condylar vein and its clinical significance. Neurosurgery 11 (Suppl 2):135-146, 2015

23. Miller M, Pearl MS, Wyse E, Olivi A, Francis HW: Decompression of the jugular bulb for enhanced infralabyrinthine access to the petroclival region: a quantitative analysis. J Neurol Surg B Skull Base 77:249-259, 2016

24. Mortimer AM, Harrington T, Steinfort B, Faulder K: Endovascular treatment of jugular bulb diverticula causing debilitating pulsatile tinnitus. J Neurointerv Surg 8:e11, 2016

25. Okudera T, Huang YP, Ohta T, Yokota A, Nakamura Y, Maehara F, et al: Development of posterior fossa dural sinuses, emissary veins, and jugular bulb: morphological and radiologic study. AJNR Am J Neuroradiol 15:1871-1883, 1994

26. Ota N, Tanikawa R, Yoshikane T, Miyama M, Miyazaki T, Kinoshita Y, et al: Surgical microanatomy of the posterior condylar emissary vein and its anatomical variations for the transcondylar fossa approach. Oper Neurosurg (Hagerstown) 13:382-391, 2017

27. Oya R, Imai T, Sato T, Uno A, Watanabe Y, Okazaki S, et al: A high jugular bulb and poor development of perivestibular aqueductal air cells are not the cause of endolymphatic hydrops in patients with Ménière's disease. Auris Nasus Larynx [epub ahead of print], 2017

28. Park JH, Son SB, Hong HP, Lee HS: A case of jugular bulb diverticulum invading the internal auditory canal. Korean J Audiol 16:39-42, 2012

29. Park JJ, Shen A, Loberg C, Westhofen M: The relationship between jugular bulb position and jugular bulb related inner ear dehiscence: a retrospective analysis. Am J Otolaryngol 36:347-351, 2015

30. Presutti L, Bonali M, Marchioni D, Pavesi G, Feletti A, Anschuetz L, et al: Expanded transcanal transpromontorial approach to the internal auditory canal and cerebellopontine angle: a cadaveric study. Acta Otorhinolaryngol Ital 37:224-230, 2017

31. Reardon MA, Raghavan P: Venous abnormalities leading to tinnitus: imaging evaluation. Neuroimaging Clin $\mathbf{N}$ Am 26:237-245, 2016

32. Roberts DS, Chen B, Slattery W: Surgical management of a high jugular bulb. Ear Nose Throat J 95:306-309, 2016

33. Sakaida H, Takeuchi K: Dehiscent high jugular bulb attached to the tympanic membrane. Ear Nose Throat J 94:210-212, 2015

34. Samii M, Alimohamadi M, Gerganov V: Endoscope-assisted retrosigmoid infralabyrinthine approach to jugular foramen tumors. J Neurosurg 124:1061-1067, 2016

35. Sasindran V, Joseph A, Abraham SS, Hiremath SB: Highriding jugular bulb: a rare entity. Indian J Otol 20:129-131, 2014

36. Schutt CA, Kveton JF: Posterior semicircular canal dehiscence secondary to jugular enlargement. Am J Otolaryngol 37:173-174, 2016

37. Shao KN, Tatagiba M, Samii M: Surgical management of high jugular bulb in acoustic neurinoma via retrosigmoid approach. Neurosurgery 32:32-37, 1993

38. Shihada R, Maimon S, Braun J, Fradis M, Luntz M: Endovascular embolization of a hemorrhagic jugular bulb diverticulum. Int J Pediatr Otorhinolaryngol 72:1445-1448, 2008

39. Signorelli F, Mahla K, Turjman F: Endovascular treatment of two concomitant causes of pulsatile tinnitus: sigmoid sinus stenosis and ipsilateral jugular bulb diverticulum. Case report and literature review. Acta Neurochir (Wien) 154:89-92, 2012

40. Singla A, Gupta T, Sahni D, Aggarwal A, Gupta A: High jugular bulb: different osseous landmarks and their clinical implications. Surg Radiol Anat 38:903-909, 2016

41. Spasic M, Trang A, Chung LK, Ung N, Thill K, Zarinkhou $\mathrm{G}$, et al: Clinical characteristics of posterior and lateral semicircular canal dehiscence. J Neurol Surg B Skull Base 76:421-425, 2015

42. Springborg JB, Poulsgaard L, Thomsen J: Nonvestibular schwannoma tumors in the cerebellopontine angle: a structured approach and management guidelines. Skull Base 18:217-227, 2008

43. Tanrivermis Sayit A, Elmali M, Kemal O, Terzi Y: Radiological, clinical and audiological evaluation of jugular bulbvestibular aqueduct dehiscence. Acta Otolaryngol 137:12211225,2017

44. Trivelato FP, Araújo JF, Dos Santos Silva R, Rezende MT, Ulhôa AC, Castro GD: Endovascular treatment of pulsatile tinnitus associated with transverse sigmoid sinus aneurysms and jugular bulb anomalies. Interv Neuroradiol 21:548-551, 2015

45. Wick CC, Hansen AR, Kutz JW Jr, Isaacson B: Endoscopic infracochlear approach for drainage of petrous apex cholesterol granulomas: a case series. Otol Neurotol 38:876-881, 2017

46. Xia Y, Zhang W, Li Y, Ma X, Liu Q, Shi J: The transotic approach for vestibular schwannoma: indications and results. Eur Arch Otorhinolaryngol 274:3041-3047, 2017

47. Yeo WX, Xu SH, Tan TY, Low YM, Yuen HW: Surgical management of pulsatile tinnitus secondary to jugular bulb or sigmoid sinus diverticulum with review of literature. Am J Otolaryngol 39:247-252, 2018

\section{Disclosures}

The authors report no conflict of interest concerning the materials or methods used in this study or the findings specified in this paper.

\section{Author Contributions}

Conception and design: Manjila, Bazil, Semaan. Acquisition of data: all authors. Analysis and interpretation of data: all authors. Drafting the article: Manjila, Bazil, Kay, Udayasankar. Critically revising the article: Manjila, Bazil, Kay, Udayasankar. Reviewed submitted version of manuscript: Manjila, Bazil, Kay. Approved the final version of the manuscript on behalf of all authors: Manjila. Study supervision: Manjila, Bazil.

\section{Supplemental Information \\ Videos}

Video 1. https://vimeo.com/268379517.

Video Abstract. https://vimeo.com/273495557.

\section{Correspondence}

Sunil Manjila: McLaren Bay Region Hospital, Bay City, MI. sunil. manjila@gmail.com. 